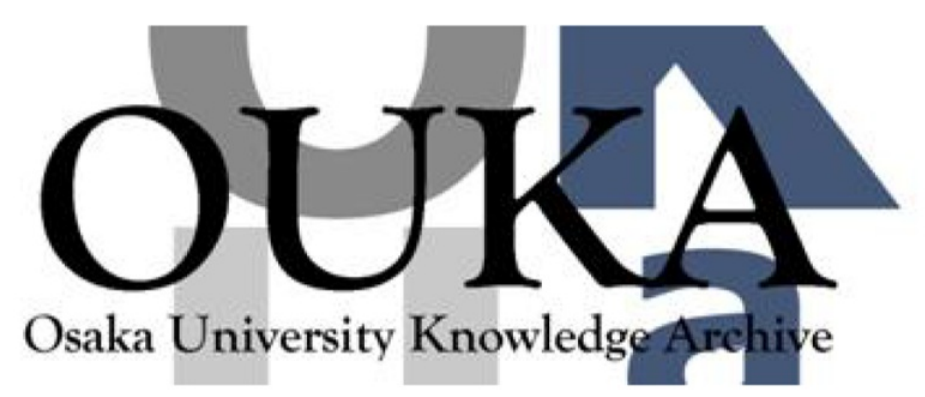

\begin{tabular}{|c|l|}
\hline Title & $\begin{array}{l}\text { Significant softening of copper nanowires } \\
\text { during electromigration studied by picosecond } \\
\text { ultrasound spectroscopy }\end{array}$ \\
\hline Author(s) & Ogi, H. ; Yamamoto, A. ; Kondou, K. et al. \\
\hline Citation & $\begin{array}{l}\text { Physical Review B - Condensed Matter and } \\
\text { Materials Physics. 82(15) p. 155436-1-p. 155436-5 }\end{array}$ \\
\hline Issue Date & $2010-10-19$ \\
\hline oaire:version & VoR \\
\hline URL & https://hdl. handle. net/11094/84180 \\
\hline rights & Copyright 2010 by the American Physical Society \\
\hline Note & \\
\hline
\end{tabular}

Osaka University Knowledge Archive : OUKA

https://ir. Library. osaka-u. ac. jp/

Osaka University 


\title{
Significant softening of copper nanowires during electromigration studied by picosecond ultrasound spectroscopy
}

\author{
H. Ogi, ${ }^{1, *}$ A. Yamamoto, ${ }^{1}$ K. Kondou, ${ }^{2}$ K. Nakano, ${ }^{2}$ K. Morita, ${ }^{1}$ N. Nakamura, ${ }^{1}$ T. Ono, ${ }^{2}$ and M. Hirao ${ }^{1}$ \\ ${ }^{1}$ Graduate School of Engineering Science, Osaka University, Toyonaka, Osaka 560-8531, Japan \\ ${ }^{2}$ Institute of Chemical Research, Kyoto University, Uji, Kyoto 611-0011, Japan
}

(Received 4 July 2010; published 19 October 2010)

\begin{abstract}
High-frequency vibrations related with copper nanowires on a silicon substrate are studied using picosecond ultrasound spectroscopy. The reflectivity change in the probe light pulse is monitored after irradiation of the specimen with the ultrafast light pulse, showing high $(\sim 75 \mathrm{GHz})$ and low $(<\sim 15 \mathrm{GHz})$ frequency vibrations, which are identified to be thickness resonance of nanowires and collective-mode resonances on the substrate, respectively. The nanowires are subjected to the current-loading test to induce electromigration. The thickness resonance frequency significantly decreases as the progress of the electromigration while the other frequencies and the resistance remain nearly unchanged. The micromechanics analysis and the vacancy diffusion theory indicate the growth of thin defects at grain boundaries for softening the nanowires.
\end{abstract}

DOI: $10.1103 /$ PhysRevB.82.155436

PACS number(s): 68.35.Iv, 78.47.J-, 78.66.-w, 62.20.D-

Mechanical spectroscopy in nanostructures has been made possible with picosecond ultrasound, including nanowires ${ }^{1-3}$ and nanodots, $1,4,5$ where the ultrafast pump light pulse causes vibrations related with the nanostructures, and the timedelayed probe light pulse detects them through the optoelastic effect. ${ }^{6}$ Intensive studies were made for identifying observed vibrations of the reflectivity change in the probe light

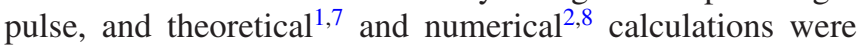
developed for explaining the vibrational frequencies. However, few studies discussed the relationship between the resonance frequencies and microstructure inside the nanostructures. In this paper, we find out significant softening of copper nanowires during electromigration using the picosecond ultrasound spectroscopy. The stiffness decreases by $\sim 20 \%$ even with a low-current density with which the electromigration failure would not occur.

Nanostructures on substrates experience the deposition procedure, which introduces defects related with vacancies. Previous studies showed that as-deposited thin films were significantly softer than the corresponding bulk materials because of the defects. ${ }^{9-11}$ Vacancies will cause diffusion with the presence of the electric field and stack at grain boundaries to form clusters and voids, known as electromigration. They can recover with low-temperature annealing procedure ${ }^{9}$ but most nanowires are used with the as-deposited condition, and the current application will cause the vacancy flow, which grows thin defects at grain boundaries, lowering the macroscopic stiffness. Thus, the stiffness will be an important characteristic for evaluating microstructure evolution in nanowires. Furthermore, the stiffness has been considered as a principal parameter affecting the electromigration behavior in confined metallic nanowires. ${ }^{12,13}$ However, evolution of the stiffness in nanowires has never been measured previously: This is the principal purpose in this paper.

The electron-beam-lithography method was used to fabricate the copper nanowire specimens on (001) Si substrates as shown in Figs. 1(a) and 1(b). 499 nanowires are aligned in parallel with a constant spacing $D$. The length, width $w$, and thickness $t$ of a nanowire were $5000 \mathrm{~nm}, 300 \mathrm{~nm}$, and $30 \mathrm{~nm}$, respectively [Fig. 1(c)]. The spacing $D$ was 800 , 1000, or
$1200 \mathrm{~nm}$. Thin aluminum lines were bonded on the two flat electrodes to apply currents in the nanowires.

We used a mode-locking titanium-sapphire pulse laser with $100 \mathrm{fs}$ pulse width. Both pump $(800 \mathrm{~nm}, 60 \mathrm{pJ})$ and probe (400 nm, $80 \mathrm{pJ}$ ) light pulses were applied perpendicularly on the specimen surface. The time resolved reflectivity
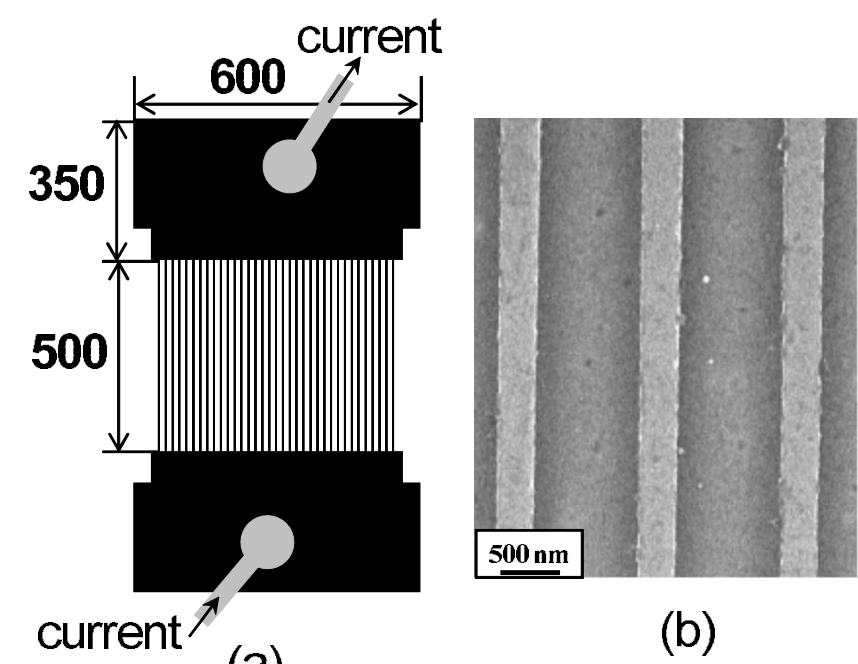

(a)

(b)

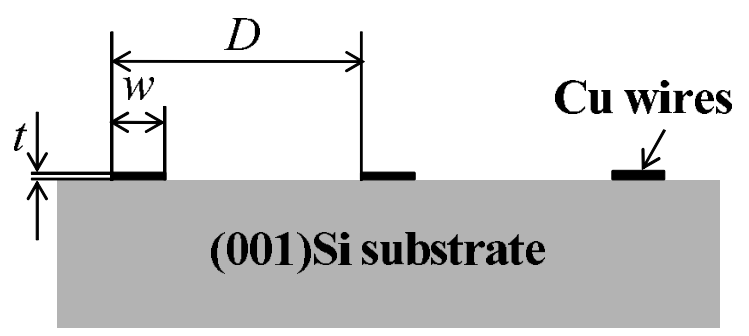

(c)

FIG. 1. (a) Schematic of the nanowire specimen fabricated on a (100) Si substrate by the electron-beam-lithography method. Numbers are dimensions in micrometer. (b) Scanning electron micrograph near the middle of the nanowire after the current-loading test. (c) Schematic of the cross section of the nanowire specimen. 

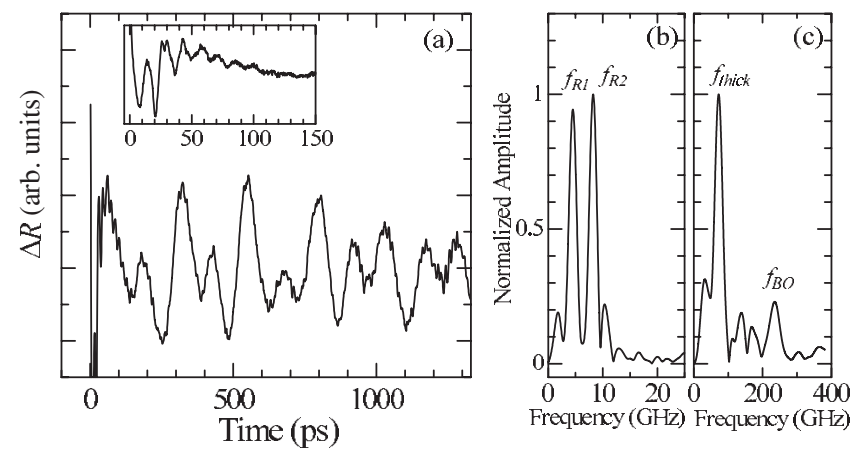

FIG. 2. (a) Time-resolved reflectivity change in the probe light, and corresponding Fourier spectra for (b) low-frequency and (c) high-frequency oscillations.

change in the probe light was measured by changing the light path of the probe light. Details of our optics appear elsewhere. ${ }^{14}$

Figure 2(a) shows the typical reflectivity changes obtained at the middle of the nanowire specimen. Just after the excitation, high-frequency oscillations are first observed (see the inlet), which are followed by low-frequency oscillations. Corresponding Fourier transform spectra [Figs. 2(b) and 2(c)] show peak frequencies at $3-10 \mathrm{GHz}$ denoted by $f_{R 1}$ and $f_{R 2}, 70-80 \mathrm{GHz}$ by $f_{\text {thick }}$, and $235 \pm 2 \mathrm{GHz}$ by $f_{B O}$. The last one corresponds to the Brillouin oscillation in $\mathrm{Si},{ }^{15}$ which is caused by interference between the surface reflected light and diffracted light by the strain pulse propagating in the $\mathrm{Si}$ substrate. Its theoretical frequency is $f_{B O}=235 \mathrm{GHz}$ at 400 $\mathrm{nm},{ }^{16}$ showing good agreement with the measurement.

The low-frequency peaks are identified as the collectivemode resonances, ${ }^{4,5}, 8$ which are dispersive surface-wave modes, as follows. Because the nanowires become vibrational sources, surface waves are generated simultaneously from individual nanowires. They can be coherent when the wavelength or its integral multiples are nearly equal to the wire spacing. Figure 3 shows the dispersion curves obtained for the nanowire specimens with different spacings of $D$ $=800,1000$, and $1200 \mathrm{~nm}$, where $k_{R}=2 \pi / D$ is the wave number. The solid line denotes the nondispersive relationship between the wave number and the frequency; its slope corresponds to the Rayleigh-wave velocity $V_{s}=5000 \mathrm{~m} / \mathrm{s}$ on $\mathrm{Si}$ (001). ${ }^{17}$ The broken lines are dispersion curves of the collective modes calculated with the effective-mass approximation near the surface: We calculated the effective mass density with $\rho_{\text {eff }}=\left(t w \rho_{w}+D h_{m} \rho_{s}\right) /\left(D h_{m}+t w\right)$ by simply considering the volume fraction of the nanowires in the propagation region. Here, $\rho_{w}$ and $\rho_{s}$ denote mass densities of the nanowire and substrate, respectively. The effective penetration depth of the $m$ th collective mode, $h_{m}$, was assumed to be $h_{m}$ $=0.15 \mathrm{D} / \mathrm{m}$. (In a homogenous material, nearly $50 \%$ kinetic energy of Rayleigh wave exists within the depth of $15 \%$ of the wavelength.) Then, the dispersion relationship is given by $\omega / k_{R}=m \sqrt{V_{s}^{2} \rho_{s} / \rho_{\text {eff }}}$. This approximation favorably explains all measurements for fundamental $\left(f_{R 1}\right)$ and second $\left(f_{R 2}\right)$ modes with only one parameter $h_{m}$, supporting that the resonance frequencies near 3-10 $\mathrm{GHz}$ represent the collective-mode resonances. We could determine the resonance frequencies within $3 \%$ error among different speci-

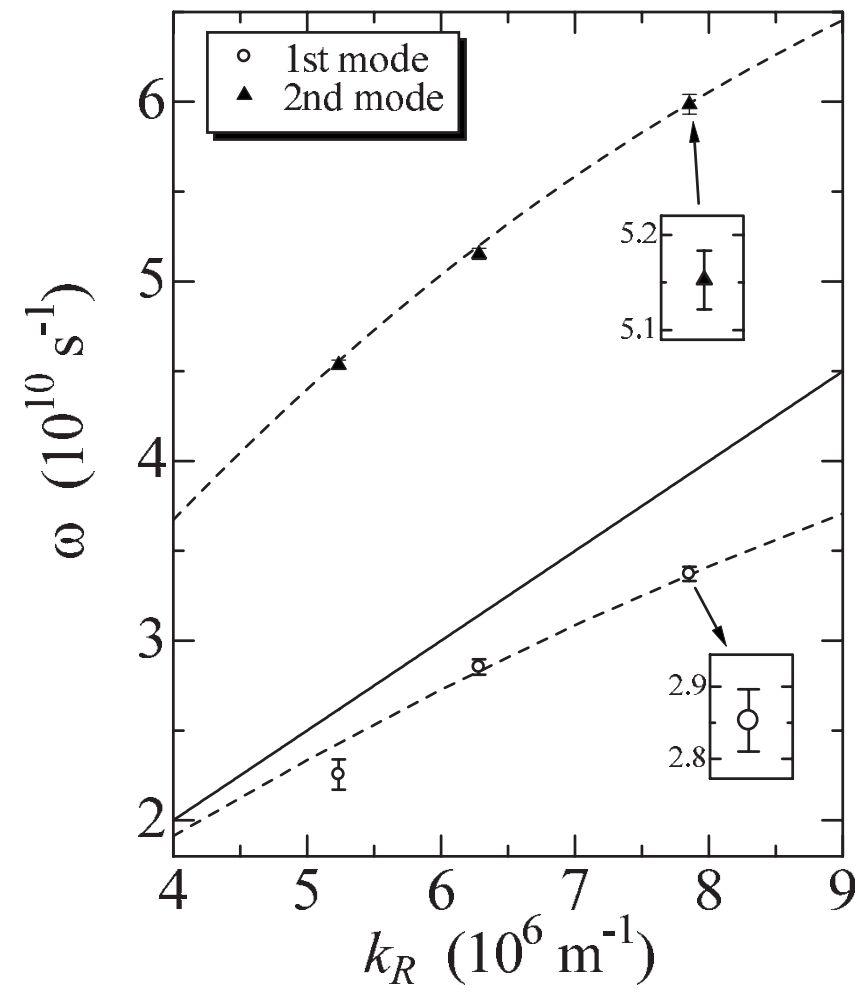

FIG. 3. Dispersion curves for collective modes. Solid line is the dispersion curve of the Rayleigh wave on (100) Si and broken lines are those calculated by the effective-mass approximation.

mens, indicating the high precision of the nanowire dimensions and high signal-to-noise ratio obtained in our optics (see inlets in Fig. 3).

The resonance frequency at $70-80 \mathrm{GHz}$ is considered as the thickness resonance in the nanowire. Because of the large aspect ratio of the cross section of the nanowire and restriction of the in-plane deformation by the substrate, the elastic constant related with the deformation in the thickness direction can be approximated by the longitudinal-wave modulus, which is $C_{w}=198.5 \mathrm{GPa}$ for polycrystalline copper. Because the acoustic impedance of $\mathrm{Cu}$ is larger than that of $\mathrm{Si}$, the reflection at the $\mathrm{Cu} / \mathrm{Si}$ interface will not cause significant phase change, and nearly free-boundary resonances occur. Thus, the resonance frequency $f_{\text {thick }}=\sqrt{C_{w} / \rho_{w}} / 2 t$ $=78.5 \mathrm{GHz}$ is obtained for the fundamental thicknessresonance mode. This value is consistent with the measured resonance frequency $(70-80 \mathrm{GHz})$, and we identify it to be the through-thickness resonance of the nanowires. [The number of grain will be small in the thickness direction but the measurement gives the averaged stiffness in the spot size of the laser beam (near $50 \mu \mathrm{m}$ in diameter), where many grains are involved, allowing the aggregated stiffness to be good approximation.] $f_{\text {thick }}$ was also independent on the wire spacing $D$, confirming that it corresponds to the throughthickness resonance.

Figure 4(a) shows changes in the resonance frequencies measured at the center region and the electric resistance as the current-loading test progresses. We applied the rectangle current pulse with the duration of $1 \mu$ s and the current density of $j=1 \mathrm{GA} / \mathrm{m}^{2}$. The repetition frequency of the pulse 

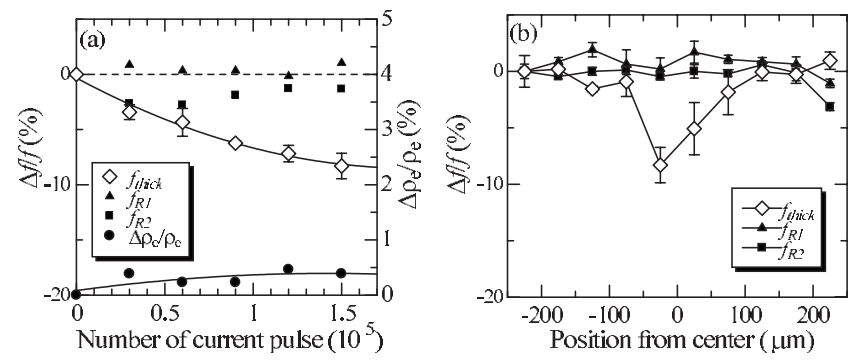

FIG. 4. (a) Changes in the resonance frequencies and electric resistance $\Delta \rho_{e}$ during the current loading test. (b) Distributions of the resonance frequencies along the nanowires after the current loading test.

was $100 \mathrm{~Hz}$. $f_{\text {thick }}$ significantly decreases whereas the collective-mode resonance frequencies remain nearly unchanged. The resistance slightly increased by $0.5 \%$. [This current density is much lower than that used in the previous study $\left(\sim 100 \mathrm{GA} / \mathrm{m}^{2}\right)$ of electromigration in copper nanowires. $\left.{ }^{18}\right] \quad f_{B O}$ was also unchanged throughout the current-loading test (not shown). The overall waveforms were identical to that of Fig. 2(a) during the current-loading test.

Figure 4(b) shows changes in the resonance frequencies along the longitudinal direction of the nanowires after the current loading test. The collective-mode resonance frequencies again remain unchanged while the through-thickness resonance frequency takes significantly lower values near the middle of the nanowires.

These observations indicate three important characteristics. First, the nanowires do not cause detachment from the substrate. Otherwise, the collective-mode resonances would have not been observed. Second, the change in the volume fraction of the defects during the electromigration should be significantly small. The spot diameters of the light pulses are about $50 \mu \mathrm{m}$, and about 50 nanowires participate in the thickness resonance. Because the through-thickness resonance frequency is highly affected by the dimensions of the nanowires, many nanowires must keep their shape so as to make the coherent vibrations observable during the currentloading test. Thus, the volume fraction of the defects will be nearly unchanged to retain the rectangle cross-section shape and dimensions. The scanning electron microscopy observation actually found no significant change in their dimensions [Fig. 1(b)]. Third, the depression in $f_{\text {thick }}$ means remarkable decrease in the stiffness of nanowires. For example, the stiffness has to be lowered by $20 \%$ to cause a $10 \%$ decrease in the resonance frequency. Also the defects are accumulated in the middle part of nanowire. The vacancy flow should be enhanced near the center of the nanowires ${ }^{19}$ because both line ends are connected to the large flat electrodes, where the temperature remains colder because of much lower current density. This effect is clearly indicated by lower $f_{\text {thick }}$ values near the center of the nanowires [Fig. 4(b)].

To explain the large decrease in the stiffness during the electromigration, we assume that initially introduced vacancies are diffused along grain boundaries, resulting in very thin defects, keeping the total volume fraction of defects nearly unchanged. Because the contribution of thin defects to

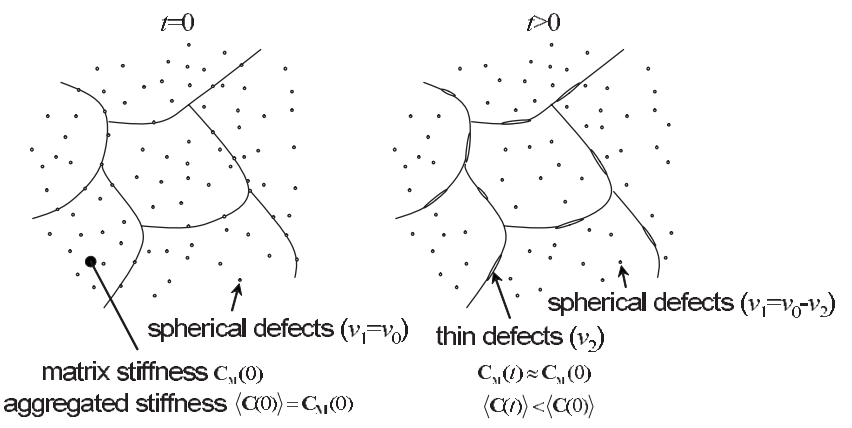

FIG. 5. Schematic of the evolution of thin defects at grain boundaries due to diffusion of spherical defects.

the stiffness decrease is much larger than that of spherical defects due to the stress concentration, ${ }^{20}$ drastic decrease in the stiffness can be expected even with a constant defect volume (Fig. 5). Based on this model, we calculate the time behavior of the stiffness during the electromigration below.

The initial microstructure consists of the polycrystalline copper and spherical defects (vacancies) with the volume fraction of $v_{0}$, and we use the micromechanics theory to calculate its macroscopic elastic constants $\mathbf{C}_{0}$ as $^{21}$

$$
\mathbf{C}_{0}=\mathbf{C}_{M}+v_{0}\left(\mathbf{C}_{d}-\mathbf{C}_{M}\right) \mathbf{A}_{d}\left(\left(1-v_{0}\right) \mathbf{I}+v_{0} \mathbf{A}_{d}\right)^{-1},
$$

where $\mathbf{A}_{d}=\left[\mathbf{S C}_{M}^{-1}\left(\mathbf{C}_{d}-\mathbf{C}_{M}\right)+\mathbf{I}\right]^{-1} . \mathbf{C}_{M}$ and $\mathbf{C}_{d}$ denote the elastic constants of the matrix and defect, respectively. $\mathbf{S}$ is the Eshelby tensor, and its nonzero components are determined by the shape of defects and Poisson's ratio of the matrix as tabulated by Mura. ${ }^{22}$ We used the aggregated elastic constants of copper using the Hill averaging method ${ }^{23}$ for $\mathbf{C}_{M}$, and the stiffness of air for $\mathbf{C}_{d}$ (nearly zero stiffness). Previous study revealed that the longitudinal-wave stiffness of deposited copper thin films is lower than that of the bulk material by a factor of $0.8-0.9 .{ }^{9}$ Here, we assume that $v_{0}$ $=0.05$, which yields the initial stiffness lower than the bulk value by $87 \%$.

The current flow causes diffusion of vacancies near the grain boundaries, growing into the thin defects with the volume fraction of $v_{2}(t)$ at a time $t$. Because of the assumption of the constant total volume of defects, the volume fraction of the spherical defects $v_{1}(t)$ decreases to $v_{0}-v_{2}(t)$ (Fig. 5). Because initially introduced spherical defects will exist uniformly and they are much smaller than the wavelength, we consider that the microstructure at $t>0$ can be regarded as a two phase composite consisting of the matrix, whose stiffness is modified by the spherical defects, and the inclusions of the thin defects. Thus, the matrix stiffness $\mathbf{C}_{M}(t)$ will increase as the electromigration progresses while the overall stiffness $\langle\mathbf{C}(t)\rangle$ should decrease more significantly because of the larger contribution of the thin defects.

The calculation of $\langle\mathbf{C}(t)\rangle$ takes three steps. First, we calculate the matrix stiffness $\mathbf{C}_{M}(t)$ with Eq. (1) by substituting $v_{1}(t)=v_{0}-v_{2}(t)$ for $v_{0}$. Second, we calculate the stiffness of such a composite that consists of the matrix with the stiffness of $\mathbf{C}_{M}(t)$ and penny-shape air inclusions for the thin defects, whose minor axes are aligned uniaxially. This composite macroscopically shows the hexagonal-symmetry stiffness, 
which can be calculated by Eq. (1) by using $\mathbf{C}_{M}(t)$ for $\mathbf{C}_{M}$ and $v_{2}(t)$ for $v_{0}$. (The Eshelby tensor for the penny-shape inclusions is used in this calculation.) Third, the hexagonalsymmetry elastic constants are averaged by the Hill averaging method to obtain the overall isotropic stiffness, involving randomly distributed penny-shape defects.

The above calculations require the key parameter $v_{2}(t)$. We estimate this using the electromigration diffusion equation. Vacancies will accumulate at a particular blocking boundary, and its rate is expressed by the one-dimensional diffusion equation along the $x$ direction: $\partial v / \partial t=D\left(\partial^{2} v / \partial x^{2}\right.$ $-\alpha \partial v / \partial x$ ), where $v$ is the vacancy concentration or the volume fraction, and $D=D_{0} \exp \left(-E / k_{B} T\right)$ is the vacancy diffusivity. $E, T$, and $k_{B}$ are the activation energy for vacancy migration, temperature, and Boltzmann constant, respectively. The parameter $\alpha=z^{*} e \rho_{e} j /\left(k_{B} T\right)$ denotes the inverse of the length for electromigration (Blech length), ${ }^{24}$ where $z^{*}, e$, and $\rho_{e}$ are effective valence, and electronic charge, resistively. Shatzkes and Lloyd derived an analytical solution of this equation for a semi-infinite conductor with a perfect blocking diffusion barrier at $x=0,{ }^{25}$ and gave the change in the vacancy concentration $v(t)$ at the boundary. We applied this equation for the volume fraction of the thin defects as

$$
v_{2}(t)=2 v_{0}\left[\beta^{2}(1+\operatorname{erf} \beta)+\frac{1}{2} \operatorname{erf} \beta+\frac{2 \beta}{\sqrt{\pi}} \exp \left(-\beta^{2}\right)\right],
$$

where $\beta=\alpha \sqrt{t D} / 2$. Figure 6 shows thus calculated evolution of the longitudinal-wave stiffness together with the experimental data. (The number of the current pulse was converted into the time using the pulse duration.) The dominant parameters were the aspect ratio of the thin defect and the activation energy for vacancy migration. Other parameters are taken from literates as listed in the caption. Calculations favorably explain the time behavior of the observed stiffness: The overall stiffness significantly decreases with time, although the matrix stiffness increases slightly. The activation energy for vacancy migration in copper is reported to be about $0.74 \mathrm{eV}$ (Ref. 26) but it is significantly lowered for diffusion along surfaces to be $0.3-0.6 \mathrm{eV} .{ }^{27}$ Our calculation requires low-activation energy as shown in Fig. 6(b), indicating dominant surface diffusion of vacancies.

One may expect some influences of the stiffness change on the collective modes. However, the contribution of the stiffness of the nanowire to the collective modes is too small to be detected through the frequency change because the lon-
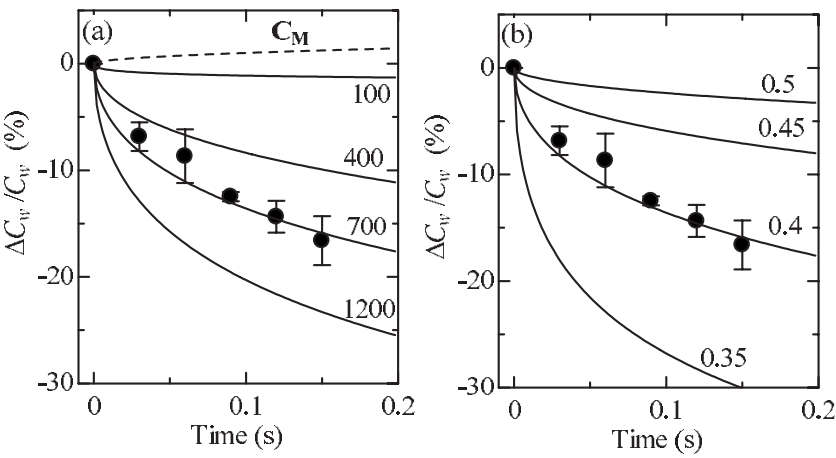

FIG. 6. Comparisons of the measured change in the longitudinal-wave stiffness (dots) with those calculated by the micromechanics model (solid lines) (a) with various aspect ratios (100-1200) of the thin defects for $E=0.4 \mathrm{eV}$ and (b) with various activation energies $(0.35-0.5 \mathrm{eV})$ at the aspect ratio of 700 . The broken line denotes the change in the matrix stiffness. The parameters used in the calculation are $D_{0}=7 \times 10^{-5} \mathrm{~m}^{2} / \mathrm{s}$ (Ref. 28), $z^{*}$ $=5$ (Ref. 29), $j=1 \mathrm{GA} / \mathrm{m}^{2}, \rho_{e}=1.68 \times 10^{-8} \Omega \mathrm{m}$, and $T=297 \mathrm{~K}$.

gitudinal and shear strains must be zero at the surface of the specimen. Thus, the nanowires cannot be strained very much and their stiffness shows negligible contribution to the collective-mode frequencies. The acceleration of the particle movement, however, can be maximum at the surface and their mass highly affects the frequencies. In the above model, the stiffness decrease is caused by rearrangement of vacancyrelated defects, remaining the volume fraction of the defects unchanged. Thus, the macroscopic mass density of the nanowire will be the same, giving no influence on the collective mode frequencies.

In summary, we performed ultrahigh-frequency vibration measurement for copper nanowires using picosecond ultrasound spectroscopy, and successfully identified vibrational modes related with collective modes (surface-acoustic-wave modes), Brillouin oscillation in the substrate, and thickness resonance of the nanowires. The thickness resonance frequency was significantly decreased by the current loading while the other frequencies remained unchanged. The remarkable stiffness decrease was considered as the principal cause for the frequency decrease, and the micromechanics calculation combined with the electromigration diffusion theory was presented to simulate the time behavior of the stiffness. The calculation indicated that vacancy diffusion along the surface and formation of thin defects to cause the significant softening of the nanowires.
*Corresponding author; ogi@me.es.osaka-u.ac.jp

${ }^{1}$ H.-N. Lin, H. J. Maris, L. B. Freund, K. Y. Lee, H. Luhn, and D.

P. Kern, J. Appl. Phys. 73, 37 (1993).

${ }^{2}$ G. A. Antonelli, H. J. Maris, S. G. Malhotra, and J. M. E. Harper, J. Appl. Phys. 91, 3261 (2002).

${ }^{3}$ A. A. Maznev, Phys. Rev. B 78, 155323 (2008).

${ }^{4}$ J.-F. Robillard, A. Devos, and I. Roch-Jeune, Phys. Rev. B 76,
092301 (2007).

${ }^{5}$ J.-F. Robillard, A. Devos, I. Roch-Jeune, and P. A. Mante, Phys. Rev. B 78, 064302 (2008).

${ }^{6}$ C. Thomsen, H. T. Grahn, H. J. Maris, and J. Tauc, Phys. Rev. B 34, 4129 (1986).

${ }^{7}$ A. A. Maznev and A. G. Every, J. Appl. Phys. 106, 113531 (2009). 
${ }^{8}$ C. Giannetti, B. Revaz, F. Banfi, M. Montagnese, G. Ferrini, F. Cilento, S. Maccalli, P. Vavassori, G. Oliviero, E. Bontempi, L. E. Depero, V. Metlushko, and F. Parmigiani, Phys. Rev. B 76, 125413 (2007).

${ }^{9}$ N. Nakamura, H. Ogi, and M. Hirao, Phys. Rev. B 77, 245416 (2008).

${ }^{10}$ N. Nakamura, H. Ogi, T. Yasui, M. Fujii, and M. Hirao, Phys. Rev. Lett. 99, 035502 (2007).

${ }^{11}$ H. Ogi, T. Shagawa, N. Nakamura, and M. Hirao, Appl. Phys. Express 2, 105001 (2009).

${ }^{12}$ M. A. Korhonen, P. Børgesen, K. N. Tu, and Che-Yu Li, J. Appl. Phys. 73, 3790 (1993)

${ }^{13}$ J. He, Z. Suo, T. N. Marieb, and J. A. Maiz, Appl. Phys. Lett. 85, 4639 (2004).

${ }^{14}$ H. Ogi, M. Fujii, N. Nakamura, T. Yasui, and M. Hirao, Phys. Rev. Lett. 98, 195503 (2007).

${ }^{15}$ A. Devos and R. Cote, Phys. Rev. B 70, 125208 (2004).

${ }^{16}$ H. Ogi, T. Shagawa, N. Nakamura, M. Hirao, H. Odaka, and N. Kihara, Phys. Rev. B 78, 134204 (2008).

${ }^{17}$ G. Crean and A. Waintal, J. Appl. Crystallogr. 19, 181 (1986).
${ }^{18}$ Q. Huang, C. M. Lilley, and R. Divan, Nanotechnology 20, 075706 (2009)

${ }^{19}$ D. G. Pierce and P. G. Brusius, Microelectron. Reliab. 37, 1053 (1997).

${ }^{20}$ H. Ogi, N. Nakamura, H. Tanei, R. Ikeda, M. Hirao, and Mikio Takemoto, Appl. Phys. Lett. 86, 231904 (2005).

${ }^{21}$ M. L. Dunn and H. Ledbetter, Acta Mater. 45, 3327 (1997).

${ }^{22}$ T. Mura, Micromechanics of Defects in Solids (Martinus Nijhoff, Boston, 1987), p. 79.

${ }^{23}$ R. Hill, J. Mech. Phys. Solids 5, 229 (1957).

${ }^{24}$ J. J. Clement and J. R. Lloyda, J. Appl. Phys. 71, 1729 (1992).

${ }^{25}$ M. Shatzkes and J. R. Lloyd, J. Appl. Phys. 59, 3890 (1986).

${ }^{26}$ R. R. Bourassa and B. Lengeler, J. Phys. F: Met. Phys. 6, 1405 (1976).

${ }^{27}$ M. Karimi, T. Tomkowski, G. Vidali, and O. Biham, Phys. Rev. B 52, 5364 (1995).

${ }^{28}$ ASM Specialty Handbook: Copper and Copper Alloys, edited by J. R. Davis (ASM International, Materials Park, 2001).

${ }^{29}$ B. Chao, S.-H. Chae, X. Zhang, K.-H. Lu, J. Im, and P. S. Ho, Acta Mater. 55, 2805 (2007). 